\title{
Adverse effects of Z-drugs for sleep disturbance in people living with dementia: a population-based cohort study
}

Kathryn Richardson ${ }^{*}$ (D), Yoon K. Loke ${ }^{1}$, Chris Fox ${ }^{1}$, lan Maidment ${ }^{2}$, Robert Howard ${ }^{3}$, Nicholas Steel ${ }^{1}$, Antony Arthur ${ }^{4}$, Penelope J. Boyd ${ }^{1}$, Clare Aldus ${ }^{4}$, Clive Ballard ${ }^{5}$ and George M. Savva ${ }^{4,6}$

\begin{abstract}
Background: Sleep disturbance is common in dementia and often treated with Z-drugs (zopiclone, zaleplon, and zolpidem). While some observational studies suggest that Z-drugs are associated with adverse events such as falls and fracture risks in older people, this has not been studied in dementia.

Methods: We used data from 27,090 patients diagnosed with dementia between January 2000 and March 2016 from the Clinical Practice Research Datalink linked to Hospital Episodes Statistics data in England. We compared adverse events for 3532 patients newly prescribed Z-drugs by time-varying dosage to (1) 1833 non-sedative-users with sleep disturbance; (2) 10,214 non-sedative-users with proximal GP consultation matched on age, sex, and antipsychotic use; and (3) 5172 patients newly prescribed benzodiazepines. We defined higher dose Z-drugs and benzodiazepines as prescriptions equivalent to $\geq 7.5 \mathrm{mg}$ zopiclone or $>5 \mathrm{mg}$ diazepam daily. Cox regression was used to estimate hazard ratios (HRs) for incident fracture, hip fracture, fall, mortality, acute bacterial infection, ischaemic stroke/transient ischaemic attack, and venous thromboembolism over a 2-year follow-up, adjusted for demographic- and health-related covariates.
\end{abstract}

Results: The mean (SD) age of patients was 83 (7.7) years, and 16,802 (62\%) were women. Of 3532 patients prescribed Z-drugs, 584 (17\%) were initiated at higher doses. For patients prescribed higher dose Z-drugs relative to non-users with sleep disturbance, the HRs (95\% confidence interval) for fractures, hip fractures, falls, and ischaemic stroke were 1.67 (1.13-2.46), 1.96 (1.16-3.31), 1.33 (1.06-1.66), and 1.88 (1.14-3.10), respectively. We observed similar associations when compared to non-sedative-users with proximal GP consultation. Minimal or inconsistent excess risks were observed at $\leq 3.75 \mathrm{mg}$ zopiclone or equivalent daily, and for mortality, infection, and venous thromboembolism. We observed no differences in adverse events for Z-drugs compared to benzodiazepines, except lower mortality rates with Z-drugs (HR [95\% confidence interval] of 0.73 [0.64-0.83]).

Conclusions: Higher dose Z-drug use in dementia is associated with increased fracture and stroke risks, similar or greater to that for higher dose benzodiazepines. Higher dose Z-drugs should be avoided, if possible, in people living with dementia, and non-pharmacological alternatives preferentially considered. Prescriptions for higher dose Z-drugs in dementia should be regularly reviewed.

(Continued on next page)

\footnotetext{
* Correspondence: kathryn.richardson@uea.ac.uk

${ }^{1}$ Norwich Medical School, University of East Anglia, Norwich NR4 7TJ, UK

Full list of author information is available at the end of the article
}

(c) The Author(s). 2020 Open Access This article is licensed under a Creative Commons Attribution 4.0 International License, which permits use, sharing, adaptation, distribution and reproduction in any medium or format, as long as you give appropriate credit to the original author(s) and the source, provide a link to the Creative Commons licence, and indicate if changes were made. The images or other third party material in this article are included in the article's Creative Commons licence, unless indicated otherwise in a credit line to the material. If material is not included in the article's Creative Commons licence and your intended use is not permitted by statutory regulation or exceeds the permitted use, you will need to obtain permission directly from the copyright holder. To view a copy of this licence, visit http://creativecommons.org/licenses/by/4.0/. The Creative Commons Public Domain Dedication waiver (http://creativecommons.org/publicdomain/zero/1.0/) applies to the data made available in this article, unless otherwise stated in a credit line to the data. 
(Continued from previous page)

Trial registration: ENCePP e-register of studies, EUPAS18006

Keywords: Dementia, Alzheimer disease, Cohort studies, Sleep initiation and maintenance disorders, Benzodiazepines, Zolpidem, Hip fractures, Accidental falls

\section{Background}

Around $60 \%$ of people living with dementia (PlwD) are affected by sleep disturbance [1, 2], including insomnia, fragmented night-time sleep, night-time wandering, or excessive day sleep [3]. Sleep disturbance affects the quality of life of PlwD and their informal carers and often leads to care home admission [4].

Benzodiazepines are frequently used for insomnia in PlwD and act by binding to gamma-aminobutyric acid, an inhibitory neurotransmitter [5]. Benzodiazepines are associated with a range of adverse side-effects including cognitive impairment, daytime sedation, tolerance, dependence, and falls [6-9]. Z-drugs (zaleplon, zopiclone, eszopiclone, and zolpidem), a class of nonbenzodiazepine gamma-aminobutyric acid agonists, have shorter half-lives and were originally believed to be safer than benzodiazepines, but their adverse effects are increasingly recognised [10, 11]. Observational studies report Z-drugs are associated with increased risks of falls, fractures, stroke, mortality, and infection in older adults [12-16]. However, these studies are generally subject to residual confounding by sleep disturbance and comorbidity. Studies have also not typically examined how the timing and dosages of Z-drugs might modify risk. More importantly, the adverse effects of Z-drugs have rarely been studied in PlwD, where these side-effects can be particularly catastrophic [17]. A recent Cochrane review found insufficient evidence to guide drug treatment of sleep problems in dementia [18], despite wide-scale prescribing [19]. In addition, the effectiveness of Z-drugs to improve sleep in older people is uncertain and considered limited [11], with cognitive behaviour therapy demonstrated to be more effective at managing insomnia than zopiclone in older adults [20].

We examined the association between first Z-drug prescription and subsequent risk of falls, fractures, mortality, infection, ischaemic stroke, and venous thromboembolism in PlwD. To reduce confounding, we compared Z-drug users with (1) non-users with sleep disturbance, (2) non-users with a proximal GP consultation, and (3) new benzodiazepine users.

\section{Methods}

\section{Study design}

We performed a series of cohort studies, using data from the Clinical Practice Research Datalink (CPRD) linked to Hospital Episode Statistics (HES), Office for National
Statistics (ONS) mortality data, and Index of Multiple Deprivation data in England. CPRD collates all diagnoses, referrals, and prescribing records for over 11.3 million patients broadly representative of the UK population [21]. Diagnosis information is electronically entered as UK Read codes [22]. HES records all diagnoses made during a hospital admission (coded using the International classification of diseases 10th revision [ICD-10]) and demographic information [23], and ONS provides date and cause of death (ICD-10 coded) [24]. The Index of Multiple Deprivation combines a number of indicators of housing, employment, income, education, and environment at the general practice level [25]. CPRD obtained ethical approval from a National Research Ethics Service Committee, allowing researchers to access anonymised data for observational studies upon approval from an Independent Scientific Advisory Committee.

\section{Study population}

We defined dementia patients by record of a dementia diagnosis in CPRD (codes in Additional file 1) or HES (ICD-10 F00-F03, G30, G31.0 or G31.1) or prescription of a cognitive enhancer (i.e. memantine, donepezil, rivastigmine, or galantamine), occurring after January 2000 when aged $\geq 55$ years. We excluded patients with $<3$ months of 'up-to-standard' (research quality) data or with severe mental illness or Down syndrome before dementia diagnosis (codes in Additional file 1) [21].

\section{Exposures}

Our primary exposure was new prescription of Z-drugs. We considered three comparator groups to reduce confounding [26]. The primary comparator was record of sleep disturbance without sedative-hypnotic prescription. Secondary comparators were (a) non-users at a proximal GP consultation and (b) new benzodiazepine users. To facilitate these comparisons, three main cohorts were constructed, with their index date as the first date after dementia diagnosis of (a) prescription for a Z-drug (World Health Organization's Anatomic Therapeutic Classification [ATC] system category N05CF), (b) prescription of any benzodiazepine (ATC N05BA or N05CD except midazolam injection), and (c) code for sleep disturbance (codes in Additional file 1) but without a concurrent sedative-hypnotic (ATC N05C or N05BA) prescription. 
Two additional cohorts were created by matching the Z-drug and BZD cohorts on age, sex, and antipsychotic use, to non-users (not prescribed sedative-hypnotics) with proximal GP consultation. We matched three nonusers to each Z-drug or benzodiazepine user, without replacement, and assigned an index date as the closest GP consultation within 1 month of the corresponding Zdrug or benzodiazepine index date. Patients could be members of different cohorts over time.

Exclusion criteria for all cohorts were $(1)<12$ months data history; (2) sedative-hypnotic prescription in the last 12 months; (3) prior diagnosis of sleep apnoea, sleep-related respiratory failure, or alcohol abuse (codes in Additional file 1); (4) prescription of multiple sedative-hypnotics; and (5) newly prescribed antipsychotics or low-dose tricyclic or related antidepressants ( $\leq 25 \mathrm{mg}$ amitriptyline or $\leq 50 \mathrm{mg}$ trazodone per day). We additionally performed a separate validation study on the accuracy of our patient selection (details in Additional file 2). In summary, GP practices confirmed dementia diagnoses in $96 \%$ of cases; however, uncertainty was raised regarding the accuracy of those identified with sleep disturbance.

To test dose-response relationships, we determined the number of daily defined doses (DDDs) of Z-drugs and benzodiazepines at each prescription. The DDD is the assumed average maintenance dose per day for a drug based on its main indication in adults. We used DDD values from the World Health Organization's Collaborating Centre for Drug Statistics Methodology (www.whocc.no/atc_ddd_index), where the DDDs for zopiclone, zolpidem, and zaleplon are $7.5 \mathrm{mg}, 10 \mathrm{mg}$, and $10 \mathrm{mg}$ per day, respectively. The British National Formulary recommends these daily doses for adults with insomnia, but to halve them for elderly patients. Missing dosing frequency data was assumed once daily, except diazepam where we applied the most common frequency for the product and quantity prescribed among the complete prescription data.

\section{Outcomes}

The selected outcomes were identified from previous studies or were priorities identified by our Patient and Public Involvement (PPI) group members or by an advisory group of healthcare professionals established to support this project. The main outcomes were, in order of importance: (1) incident (a) fracture in any location, (b) hip fracture, and (c) forearm/wrist/hand fracture; (2) incident fall; (3) mortality; (4) acute bacterial infection; (5) ischaemic stroke/transient ischaemic attack; and (6) venous thromboembolism. These were identified via first mention of a relevant Read code in CPRD or ICD-10 code in HES or ONS (codes in Additional file 3). We also examined further healthcare utilisation outcomes:
(7) number of (a) hospital admissions and (b) GP consultations and (8) new prescription of (a) antipsychotics, (b) antidepressants, and (c) antibiotics.

\section{Covariates}

We considered as potential confounders variables suspected to be linked to dementia, sleep disturbance, benzodiazepine or Z-drug use, or the outcomes examined. They were measured on the index date and covered domains of demographics, health behaviours, dementia subtype, proxies for dementia severity, proxies for sleep disturbance severity, comorbidities, recent medical history (e.g. GP consultations, hospital admissions, falls, fractures, infections, immunisations, body mass index [BMI], systolic blood pressure), and concurrent prescriptions (details in Additional file 4).

\section{Statistical analysis}

The primary analysis estimated the association between new prescription of sleep disturbance medication and incidence of each outcome, compared to other groups. We followed patients until the earliest of death, leaving the GP practice, last data extraction, new sedative-hypnotic or antipsychotic prescription, 2 years post-index date, or 31 March 2016. Z-drug and benzodiazepine new users were also censored 90 days following their last Z-drug/ benzodiazepine prescription. Matched patients were additionally censored at the censoring date of their corresponding case. Specific exclusion criteria applied at the index date to reduce the chance of repeated coding of the same event are described in Additional file 5 table S1.

Cox proportional hazards regression was used for binary outcomes. We used robust standard errors to account for the correlation due to repeat measurements in some patients [27]. The proportional hazards assumption was checked using Schoenfeld residuals [28]. Negative binomial regression was used to model the number of hospital admissions and GP consultations. Estimates were adjusted for age and sex and all covariates in Additional file 4. Age, BMI, systolic blood pressure, duration since dementia diagnosis, index date, and number of prior GP consultations were modelled using restricted cubic splines (with five knots) to allow non-linear effects [29]. We included an interaction between sex and BMI, due to known sex differences in the relationship between BMI and fracture risk [30]. Absolute risk differences of adverse events and numbers needed to harm (NNH) were estimated using standard formulae for time to event analysis [31].

We examined the average daily Z-drug dose over follow-up, but in post hoc secondary analysis, to reflect changes in dose, we examined time-varying daily DDDs. In the sensitivity analysis, we excluded those with record 
of $>6 \mathrm{~h}$ sleep per night from the sleep-disturbance comparator group (see validation study in Additional file 2 for more detail). This was to increase the chance that the sleep-disturbance group had more comparable insomnia to the Z-drug group. Finally, in the comparison of Z-drug to benzodiazepine new users, we restricted to benzodiazepines likely prescribed for sleep disturbance (loprazolam, lormetazepam, nitrazepam, temazepam, or other benzodiazepines with dosing instructions to take only at night or with a concurrent record of sleep disturbance).

Multiple imputation by chained equations was used to impute missing values of BMI, smoking, alcohol use, residence, ethnicity, and blood pressure (see Additional file 4 for details) [32]. To account for multiple outcomes tested, we used the Benjamini-Hochberg procedure to control the false discovery rate at $<5 \%$ for each analysis [33]. Stata version 15.1 was used throughout.

\section{Results}

There were 51,117 eligible dementia patients with $\geq 12$ month data history in the linked CPRD-HES database (Additional file 5 figure S1). Of these, 3532 and 5172 patients were newly prescribed Z-drugs or benzodiazepines, respectively, and met our inclusion criteria. Zdrug and benzodiazepine new users were matched to 10 , 214 and 15,174 non-users, respectively. Finally, 1833 dementia patients had recorded sleep disturbance, but without prescription of sedative-hypnotics.

\section{Patient characteristics}

The mean (SD) age of patients at index date was 83 (7.7) years, and 16,802 (62\%) were women. Patients were registered with their GP for a median (IQR) of 19 (11-32) years and diagnosed with dementia for a median (IQR) of $12(4-25)$ months.

The patient cohorts were similar across measured characteristics (Table 1 with missing data described in Additional file 5 table S2). Recent hospital admissions were more likely among Z-drug users. Concurrent antipsychotics and antidepressants and previous benzodiazepine or Z-drug use was more likely among benzodiazepine and Z-drug users. Benzodiazepine users more frequently lived in care homes and had agitation/ psychosis and anxiety and dementia for longer. Those with sleep disturbance (no sedative-hypnotics) were more likely from a deprived neighbourhood, consume alcohol, and have urinary incontinence and insomnia history before dementia. Finally, the non-users with proximal GP consultation had more recent GP consultations and fewer recent falls and fractures.

Of 3532 patients prescribed Z-drugs, 3358 (95\%) were prescribed zopiclone, with 2801 (83\%) prescribed 3.75 mg daily on the index date. For 598 (17\%), the prescription instructions were 'pro re nata' (PRN/as needed). Of 5172 patients prescribed benzodiazepines, the most common were diazepam $(n=2077,40 \%)$, lorazepam $(n=1669,32 \%)$, and temazepam $(n=1168,23 \%)$. Patients were followed up for a median (IQR) of 3.5 (3.0-10.3) months and mainly censored due to no further Z-drug or benzodiazepine prescriptions. See Additional file 5 table S3 for initial and follow-up doses.

\section{Falls and fractures}

We estimated HRs (95\% CI) of $1.32(0.99-1.75)$ and 1.34 (1.08-1.67) for Z-drugs and fracture compared to sleep disturbance (without sedative-hypnotics) and non-use with proximal GP consultation, respectively (Table 2). For hip fracture, the HRs $(95 \% \mathrm{CI})$ were $1.38(0.92-2.06)$ and $1.59(1.15-2.19)$ for Z-drugs compared to sleep disturbance and non-use with proximal GP consultation, respectively. Z-drug use was associated with increased falls compared to nonuse with proximal GP consultation (HR 1.43, 95\% CI 1.26-1.62), but not compared to sleep disturbance (HR 1.02, 95\% CI 0.87-1.21). New benzodiazepine use was associated with increased fractures and falls compared to non-use with proximal GP consultation, but the HR $(95 \% \mathrm{CI})$ for hip fractures was 1.17 (0.871.57) (Additional file 5 table S4). There were no large differences in fall and fracture rates between new Zdrug and benzodiazepine users (Table 2).

There was evidence of differing associations with the outcomes according to the prescribed daily dose of Zdrugs or benzodiazepines (Table 3 and Additional file 5 table S5). Compared to sleep disturbance without sedative-hypnotics, the adjusted HRs (95\% CI) for fractures, hip fractures, and falls for Z-drug prescriptions equivalent to $\geq 7.5 \mathrm{mg}$ zopiclone daily were 1.67 (1.132.46), 1.96 (1.16-3.31), and 1.33 (1.06-1.66), respectively. The adjusted HRs (95\% CI) for Z-drug prescriptions equivalent to $\leq 3.75 \mathrm{mg}$ zopiclone daily and fractures, hip fractures, and falls were 1.22 (0.90-1.66), $1.21(0.78-1.90)$, and $0.95(0.80-1.13)$, respectively. Similar associations were observed when compared to nonusers with proximal GP consultation.

\section{Absolute risks}

The use of zopiclone at $\geq 7.5 \mathrm{mg}$ or equivalent is associated with absolute annual risks of fracture of $12.4 \%$ (compared to $7.6 \%$ in the sleep disturbance cohort). For hip fracture, the corresponding figures are $6.6 \%$ annual risk associated with zopiclone at $\geq 7.5 \mathrm{mg}$ or equivalent compared to $3.4 \%$. This is equivalent to $\mathrm{NNH}$ of 21 and 32, and extra cases per 1000 treated of 48 and 32 for fractures and hip fractures, respectively. 
Table 1 Characteristics of patients with dementia prescribed Z-drugs and benzodiazepines and comparison cohorts

\begin{tabular}{|c|c|c|c|c|c|c|c|c|c|c|}
\hline \multirow[b]{2}{*}{ Characteristic } & \multicolumn{2}{|c|}{$\begin{array}{l}\text { Z-drug } \\
(n=3532)\end{array}$} & \multicolumn{2}{|c|}{$\begin{array}{l}\text { Sleep disturbance, no sedative-hypnotic } \\
(n=1833)\end{array}$} & \multicolumn{2}{|c|}{$\begin{array}{l}\text { No Z-drug, } \\
\text { proximal GP } \\
\text { consultation } \\
(n=10,214)\end{array}$} & \multicolumn{2}{|c|}{$\begin{array}{l}\text { Benzodiazepine } \\
(n=5172)\end{array}$} & \multicolumn{2}{|c|}{$\begin{array}{l}\text { No benzodiazepine } \\
\text { proximal GP } \\
\text { consultation } \\
(n=15,174)\end{array}$} \\
\hline & $\bar{n}$ & $\%$ & $\bar{n}$ & $\%$ & $n$ & $\%$ & $n$ & $\%$ & $\bar{n}$ & $\%$ \\
\hline Women & 2087 & $59 \%$ & 1145 & $62 \%$ & 6074 & $59 \%$ & 3222 & $62 \%$ & 9491 & $63 \%$ \\
\hline Age, years ${ }^{\mathrm{a}}$ & 82.9 & 7.7 & 83.1 & 7.1 & 83.1 & 7.5 & 82.5 & 7.8 & 82.6 & 7.8 \\
\hline White ethnicity ${ }^{b}$ & 3072 & $87 \%$ & 1539 & $84 \%$ & 8816 & $86 \%$ & 4470 & $86 \%$ & 13,042 & $86 \%$ \\
\hline Care home $^{\mathrm{b}}$ & 892 & $25 \%$ & 434 & $24 \%$ & 2108 & $21 \%$ & 1509 & $29 \%$ & 2979 & $20 \%$ \\
\hline Lives alone $^{\mathrm{b}}$ & 760 & $22 \%$ & 545 & $30 \%$ & 2848 & $28 \%$ & 993 & $19 \%$ & 4785 & $32 \%$ \\
\hline GP practice area IMD quintile ${ }^{a}$ & 3.2 & 1.4 & 3.4 & 1.4 & 3.1 & 1.4 & 3.2 & 1.4 & 3.1 & 1.4 \\
\hline Current smoker $^{\mathrm{b}}$ & 312 & $9 \%$ & 142 & $8 \%$ & 722 & $7 \%$ & 355 & $7 \%$ & 1124 & $7 \%$ \\
\hline Ex-smoker ${ }^{b}$ & 765 & $22 \%$ & 428 & $23 \%$ & 2226 & $22 \%$ & 1163 & $22 \%$ & 3322 & $22 \%$ \\
\hline Alcohol drinker ${ }^{b}$ & 715 & $20 \%$ & 489 & $27 \%$ & 2142 & $21 \%$ & 1127 & $22 \%$ & 3297 & $22 \%$ \\
\hline Body mass index ${ }^{a, b}$ & 24.9 & 4.9 & 24.5 & 4.6 & 24.9 & 4.8 & 24.5 & 4.8 & 24.8 & 4.9 \\
\hline Systolic blood pressure & 133.5 & 19.0 & 133.9 & 18.8 & 134.3 & 19.1 & 133.6 & 18.9 & 134.5 & 18.8 \\
\hline \multicolumn{11}{|l|}{ Dementia } \\
\hline $\begin{array}{l}\text { Months since dementia } \\
\text { diagnosis }^{c}\end{array}$ & 11.4 & $\begin{array}{l}3.6- \\
26.2\end{array}$ & 11.0 & $3.8-24.1$ & 13.5 & $\begin{array}{l}5.2- \\
27.4\end{array}$ & 15.6 & $5.1-32.4$ & 10.3 & $3.7-22.0$ \\
\hline \multicolumn{11}{|l|}{ Dementia subtype } \\
\hline Alzheimer's disease & 1355 & $38 \%$ & 746 & $41 \%$ & 4224 & $41 \%$ & 2140 & $41 \%$ & 6612 & $44 \%$ \\
\hline Vascular dementia & 940 & $27 \%$ & 487 & $27 \%$ & 2773 & $27 \%$ & 1375 & $27 \%$ & 4046 & $27 \%$ \\
\hline Other/mixed dementia & 409 & $12 \%$ & 192 & $10 \%$ & 1013 & $10 \%$ & 600 & $12 \%$ & 1377 & $9 \%$ \\
\hline Unspecified dementia & 828 & $23 \%$ & 403 & $22 \%$ & 2184 & $21 \%$ & 1057 & $20 \%$ & 3139 & $21 \%$ \\
\hline Agitation/psychosis history & 619 & $18 \%$ & 409 & $22 \%$ & 1261 & $12 \%$ & 1363 & $26 \%$ & 1341 & $9 \%$ \\
\hline End of life care & 197 & $6 \%$ & 77 & $4 \%$ & 564 & $6 \%$ & 416 & $8 \%$ & 726 & $5 \%$ \\
\hline \multicolumn{11}{|l|}{ Sleep disturbance } \\
\hline $\begin{array}{l}\text { Sleep disturbance pre- } \\
\text { dementia }\end{array}$ & 825 & $23 \%$ & 615 & $34 \%$ & 1982 & $19 \%$ & 1064 & $21 \%$ & 2918 & $19 \%$ \\
\hline $\begin{array}{l}\text { History of benzodiazepine } \\
\text { use }\end{array}$ & 869 & $25 \%$ & 222 & $12 \%$ & 1376 & $13 \%$ & 982 & $19 \%$ & 2009 & $13 \%$ \\
\hline History of Z-drug use & 304 & $9 \%$ & 113 & $6 \%$ & 597 & $6 \%$ & 481 & $9 \%$ & 852 & $6 \%$ \\
\hline \multicolumn{11}{|l|}{ Medical history in the past year } \\
\hline Falls & 1003 & $28 \%$ & 542 & $30 \%$ & 2165 & $21 \%$ & 1241 & $24 \%$ & 3359 & $22 \%$ \\
\hline Fractures & 360 & $10 \%$ & 131 & $7 \%$ & 657 & $6 \%$ & 409 & $8 \%$ & 1071 & $7 \%$ \\
\hline Dizziness/unsteadiness & 214 & $6 \%$ & 127 & $7 \%$ & 652 & $6 \%$ & 294 & $6 \%$ & 817 & $5 \%$ \\
\hline Faints/syncope & 184 & $5 \%$ & 120 & $7 \%$ & 533 & $5 \%$ & 357 & $7 \%$ & 712 & $5 \%$ \\
\hline $\begin{array}{l}\text { Urinary tract infection/acute } \\
\text { LRTI }\end{array}$ & 928 & $26 \%$ & 423 & $23 \%$ & 2054 & $20 \%$ & 1242 & $24 \%$ & 3114 & $21 \%$ \\
\hline Influenza vaccination & 2480 & $70 \%$ & 1340 & $73 \%$ & 7747 & $76 \%$ & 3739 & $72 \%$ & 11,350 & $75 \%$ \\
\hline Pneumonia vaccination & 166 & $5 \%$ & 103 & $6 \%$ & 506 & $5 \%$ & 234 & $5 \%$ & 773 & $5 \%$ \\
\hline Physician consultations ${ }^{a}$ & 12.5 & 10.3 & 11.2 & 9.4 & 14.9 & 12.7 & 12.9 & 10.7 & 13.7 & 12.0 \\
\hline Hospital admissions ${ }^{\mathrm{a}}$ & 1.3 & 3.0 & 1.0 & 2.2 & 0.9 & 1.6 & 1.1 & 1.9 & 0.9 & 2.3 \\
\hline \multicolumn{11}{|l|}{ Comorbidities } \\
\hline Depression & 894 & $25 \%$ & 493 & $27 \%$ & 2633 & $26 \%$ & 1512 & $29 \%$ & 3723 & $25 \%$ \\
\hline Depression symptoms & 692 & $20 \%$ & 368 & $20 \%$ & 1898 & $19 \%$ & 1175 & $23 \%$ & 2804 & $18 \%$ \\
\hline Anxiety & 576 & $16 \%$ & 311 & $17 \%$ & 1648 & $16 \%$ & 1204 & $23 \%$ & 2451 & $16 \%$ \\
\hline Anxiety symptoms & 446 & $13 \%$ & 261 & $14 \%$ & 1181 & $12 \%$ & 877 & $17 \%$ & 1751 & $12 \%$ \\
\hline
\end{tabular}


Table 1 Characteristics of patients with dementia prescribed Z-drugs and benzodiazepines and comparison cohorts (Continued)

\begin{tabular}{|c|c|c|c|c|c|c|c|c|c|c|}
\hline \multirow[b]{2}{*}{ Characteristic } & \multicolumn{2}{|c|}{$\begin{array}{l}\text { Z-drug } \\
(n=3532)\end{array}$} & \multicolumn{2}{|c|}{$\begin{array}{l}\text { Sleep disturbance, no sedative-hypnotic } \\
(n=1833)\end{array}$} & \multicolumn{2}{|c|}{$\begin{array}{l}\text { No Z-drug, } \\
\text { proximal GP } \\
\text { consultation } \\
(n=10,214)\end{array}$} & \multicolumn{2}{|c|}{$\begin{array}{l}\text { Benzodiazepine } \\
(n=5172)\end{array}$} & \multicolumn{2}{|c|}{$\begin{array}{l}\text { No benzodiazepine, } \\
\text { proximal GP } \\
\text { consultation } \\
(n=15,174)\end{array}$} \\
\hline & $n$ & $\%$ & $n$ & $\%$ & $n$ & $\%$ & $n$ & $\%$ & $n$ & $\%$ \\
\hline Parkinson's disease & 208 & $6 \%$ & 109 & $6 \%$ & 530 & $5 \%$ & 290 & $6 \%$ & 723 & $5 \%$ \\
\hline Urinary incontinence & 520 & $15 \%$ & 465 & $25 \%$ & 1640 & $16 \%$ & 841 & $16 \%$ & 2273 & $15 \%$ \\
\hline Benign prostatic hyperplasia & 361 & $10 \%$ & 175 & $10 \%$ & 1075 & $11 \%$ & 487 & $9 \%$ & 1474 & $10 \%$ \\
\hline Asthma & 366 & $10 \%$ & 165 & $9 \%$ & 1056 & $10 \%$ & 517 & $10 \%$ & 1590 & $10 \%$ \\
\hline Cancer & 743 & $21 \%$ & 313 & $17 \%$ & 2114 & $21 \%$ & 1002 & $19 \%$ & 3074 & $20 \%$ \\
\hline COPD & 266 & $8 \%$ & 147 & $8 \%$ & 782 & $8 \%$ & 369 & $7 \%$ & 1209 & $8 \%$ \\
\hline Osteoporosis & 417 & $12 \%$ & 215 & $12 \%$ & 1224 & $12 \%$ & 581 & $11 \%$ & 1885 & $12 \%$ \\
\hline $\begin{array}{l}\text { Other muscleroskeletal } \\
\text { conditions }\end{array}$ & 448 & $13 \%$ & 248 & $14 \%$ & 1395 & $14 \%$ & 664 & $13 \%$ & 2036 & $13 \%$ \\
\hline $\begin{array}{l}\text { Osteoarthritis/rheumatoid } \\
\text { arthritis }\end{array}$ & 1426 & $40 \%$ & 756 & $41 \%$ & 3980 & $39 \%$ & 2063 & $40 \%$ & 5906 & $39 \%$ \\
\hline Other joint conditions & 2901 & $82 \%$ & 1537 & $84 \%$ & 8463 & $83 \%$ & 4293 & $83 \%$ & 12,615 & $83 \%$ \\
\hline Headache/migraine & 720 & $20 \%$ & 363 & $20 \%$ & 2020 & $20 \%$ & 1101 & $21 \%$ & 2955 & $19 \%$ \\
\hline Back/neck pain & 1959 & $55 \%$ & 1009 & $55 \%$ & 5640 & $55 \%$ & 2910 & $56 \%$ & 8380 & $55 \%$ \\
\hline $\begin{array}{l}\text { Age-related macular } \\
\text { degeneration }\end{array}$ & 189 & $5 \%$ & 115 & $6 \%$ & 648 & $6 \%$ & 348 & $7 \%$ & 923 & $6 \%$ \\
\hline Cataract & 992 & $28 \%$ & 547 & $30 \%$ & 2940 & $29 \%$ & 1400 & $27 \%$ & 4284 & $28 \%$ \\
\hline Glaucoma & 356 & $10 \%$ & 186 & $10 \%$ & 1011 & $10 \%$ & 445 & $9 \%$ & 1459 & $10 \%$ \\
\hline Retinal disorder & 295 & $8 \%$ & 138 & $8 \%$ & 944 & $9 \%$ & 415 & $8 \%$ & 1390 & $9 \%$ \\
\hline Diabetes & 531 & $15 \%$ & 245 & $13 \%$ & 1712 & $17 \%$ & 718 & $14 \%$ & 2483 & $16 \%$ \\
\hline Hyperlipidaemia & 470 & $13 \%$ & 269 & $15 \%$ & 1443 & $14 \%$ & 754 & $15 \%$ & 2152 & $14 \%$ \\
\hline Hypertension & 1822 & $52 \%$ & 1006 & $55 \%$ & 5736 & $56 \%$ & 2762 & $53 \%$ & 8521 & $56 \%$ \\
\hline $\begin{array}{l}\text { Stroke/transient ischaemic } \\
\text { attack }\end{array}$ & 783 & $22 \%$ & 397 & $22 \%$ & 2177 & $21 \%$ & 1091 & $21 \%$ & 3022 & $20 \%$ \\
\hline Myocardial infarction & 310 & $9 \%$ & 172 & $9 \%$ & 909 & $9 \%$ & 433 & $8 \%$ & 1307 & $9 \%$ \\
\hline Heart failure & 314 & $9 \%$ & 182 & $10 \%$ & 988 & $10 \%$ & 444 & $9 \%$ & 1350 & $9 \%$ \\
\hline Atrial fibrillation & 528 & $15 \%$ & 260 & $14 \%$ & 1666 & $16 \%$ & 775 & $15 \%$ & 2365 & $16 \%$ \\
\hline Angina & 532 & $15 \%$ & 308 & $17 \%$ & 1646 & $16 \%$ & 811 & $16 \%$ & 2263 & $15 \%$ \\
\hline Venous thromboembolism & 236 & $7 \%$ & 123 & $7 \%$ & 737 & $7 \%$ & 341 & $7 \%$ & 1029 & $7 \%$ \\
\hline \multicolumn{11}{|l|}{ Prescriptions in the last 90 days } \\
\hline $\begin{array}{l}\text { Anticholinesterase/ } \\
\text { memantine }\end{array}$ & 850 & $24 \%$ & 385 & $21 \%$ & 2417 & $24 \%$ & 1237 & $24 \%$ & 3662 & $24 \%$ \\
\hline Antipsychotic & 811 & $23 \%$ & 371 & $20 \%$ & 1586 & $16 \%$ & 1125 & $22 \%$ & 851 & $6 \%$ \\
\hline SSRI antidepressant & 763 & $22 \%$ & 352 & $19 \%$ & 1855 & $18 \%$ & 1174 & $23 \%$ & 2616 & $17 \%$ \\
\hline Tricyclic antidepressant & 363 & $10 \%$ & 192 & $10 \%$ & 835 & $8 \%$ & 596 & $12 \%$ & 1748 & $12 \%$ \\
\hline Other antidepressant & 294 & $8 \%$ & 153 & $8 \%$ & 575 & $6 \%$ & 483 & $9 \%$ & 771 & $5 \%$ \\
\hline Antiepileptic & 241 & $7 \%$ & 110 & $6 \%$ & 610 & $6 \%$ & 424 & $8 \%$ & 825 & $5 \%$ \\
\hline Analgesic & 1730 & $49 \%$ & 808 & $44 \%$ & 4107 & $40 \%$ & 2338 & $45 \%$ & 5805 & $38 \%$ \\
\hline Inhaled corticosteroid & 191 & $5 \%$ & 96 & $5 \%$ & 507 & $5 \%$ & 196 & $4 \%$ & 742 & $5 \%$ \\
\hline Lipid regulating medication & 1217 & $34 \%$ & 630 & $34 \%$ & 3492 & $34 \%$ & 1644 & $32 \%$ & 5324 & $35 \%$ \\
\hline Diuretic & 1188 & $34 \%$ & 633 & $35 \%$ & 3322 & $33 \%$ & 1456 & $28 \%$ & 4779 & $31 \%$ \\
\hline Beta blocker & 645 & $18 \%$ & 328 & $18 \%$ & 1907 & $19 \%$ & 850 & $16 \%$ & 2771 & $18 \%$ \\
\hline ACE inhibitor & 709 & $20 \%$ & 379 & $21 \%$ & 2222 & $22 \%$ & 991 & $19 \%$ & 3357 & $22 \%$ \\
\hline
\end{tabular}


Table 1 Characteristics of patients with dementia prescribed Z-drugs and benzodiazepines and comparison cohorts (Continued)

\begin{tabular}{|c|c|c|c|c|c|c|c|c|c|c|}
\hline \multirow[b]{2}{*}{ Characteristic } & \multicolumn{2}{|c|}{$\begin{array}{l}\text { Z-drug } \\
(n=3532)\end{array}$} & \multicolumn{2}{|c|}{$\begin{array}{l}\text { Sleep disturbance, no sedative-hypnotic } \\
(n=1833)\end{array}$} & \multicolumn{2}{|c|}{$\begin{array}{l}\text { No Z-drug, } \\
\text { proximal GP } \\
\text { consultation } \\
(n=10,214)\end{array}$} & \multicolumn{2}{|c|}{$\begin{array}{l}\text { Benzodiazepine } \\
(n=5172)\end{array}$} & \multicolumn{2}{|c|}{$\begin{array}{l}\text { No benzodiazepine, } \\
\text { proximal GP } \\
\text { consultation } \\
(n=15,174)\end{array}$} \\
\hline & $n$ & $\%$ & $n$ & $\%$ & $n$ & $\%$ & $n$ & $\%$ & $n$ & $\%$ \\
\hline $\begin{array}{l}\text { Angiotensin II receptor } \\
\text { antagonist }\end{array}$ & 235 & $7 \%$ & 124 & $7 \%$ & 724 & $7 \%$ & 330 & $6 \%$ & 1123 & $7 \%$ \\
\hline Calcium channel blocker & 643 & $18 \%$ & 339 & $18 \%$ & 1947 & $19 \%$ & 849 & $16 \%$ & 2980 & $20 \%$ \\
\hline Anticoagulant & 204 & $6 \%$ & 103 & $6 \%$ & 739 & $7 \%$ & 295 & $6 \%$ & 1056 & $7 \%$ \\
\hline Antiplatelet & 1603 & $45 \%$ & 878 & $48 \%$ & 4390 & $43 \%$ & 2171 & $42 \%$ & 6439 & $42 \%$ \\
\hline Cardiac glycoside & 281 & $8 \%$ & 155 & $8 \%$ & 768 & $8 \%$ & 345 & $7 \%$ & 1032 & $7 \%$ \\
\hline NSAID & 352 & $10 \%$ & 156 & $9 \%$ & 815 & $8 \%$ & 468 & $9 \%$ & 1206 & $8 \%$ \\
\hline Bisphosphonate & 375 & $11 \%$ & 194 & $11 \%$ & 1074 & $11 \%$ & 477 & $9 \%$ & 1618 & $11 \%$ \\
\hline Calcium/vitamin D & 677 & $19 \%$ & 377 & $21 \%$ & 1854 & $18 \%$ & 888 & $17 \%$ & 2754 & $18 \%$ \\
\hline Antibiotic (in last 30 days) & 1098 & $31 \%$ & 567 & $31 \%$ & 2590 & $25 \%$ & 1370 & $26 \%$ & 3640 & $24 \%$ \\
\hline
\end{tabular}

Abbreviations: ACE angiotensin-converting enzyme, COPD chronic obstructive pulmonary disease, IMD Index of Multiple Deprivation, GP general practitioner, LRTI lower respiratory tract infection, NSAID nonsteroidal anti-inflammatory drug, SSRI selective serotonin reuptake inhibitor ${ }^{a}$ Mean (standard deviation)

${ }^{\mathrm{b}}$ Characteristic contains missing data as described in Additional file 5 table S2

${ }^{\mathrm{C}}$ Median (inter-quartile range)

\section{Mortality, infection, and cardiovascular outcomes}

Although Z-drug use was associated with greater mortality compared to those with sleep disturbance (HR 1.38, 95\% CI 1.14-1.66), there was no strong evidence of excess risk compared to non-users with a proximal GP consultation (HR 1.08, 95\% CI 0.94-1.23) (Table 2). Further, the associations with mortality seemed unrelated to dose (Table 3). Z-drug prescription was associated with less mortality than benzodiazepines (HR 0.73, 95\% CI $0.64-0.83)$.

We did not detect any strong associations between new Z-drug prescription and greater infection or venous thromboembolism rates, compared either to the nonusers or to the benzodiazepine users.

When examining new Z-drug prescription overall, we did not detect strong associations with incident stroke rates (HR 1.14 [95\% CI 0.86-1.50], compared to nonusers with proximal GP consultation). However, higher dose ( $\geq 7.5 \mathrm{mg}$ zopiclone or equivalent) Z-drugs were associated with more ischaemic strokes (HR 1.88 [95\% CI $1.14-3.10]$ and 1.90 [1.30-2.79] compared to sleep disturbance and non-users with proximal GP consultation). The association for higher dose Z-drugs appeared greater than that for higher dose $(>5 \mathrm{mg}$ diazepam or equivalent) benzodiazepine use, with a HR (95\% CI) for higher dose benzodiazepine and stroke of 1.37 (0.912.08) compared to non-use with proximal GP consultation (Additional file 5 table S5).

\section{Absolute risks}

The use of zopiclone at $\geq 7.5 \mathrm{mg}$ or equivalent is associated with absolute annual risks of stroke of $8.1 \%$ (compared to $4.4 \%$ in the sleep disturbance cohort). This is equivalent to an $\mathrm{NNH}$ of 27 and 37 extra cases per 1000 treated.

\section{Additional medication and healthcare utilisation}

The adjusted rate ratios (95\% CI) for hospital visits for Z-drug users were 1.26 (1.13-1.40), compared to sleep disturbance, and 1.17 (1.07-1.27), compared to non-use with proximal GP consultation. The rates between Zdrugs and benzodiazepines were similar. For GP consultations, the corresponding rate ratios were 1.17 (1.12$1.23)$ and 1.07 (1.04-1.11), respectively. However, when analysed by time-varying prescribed dose, more frequent hospital admissions and GP consultations were generally only observed for higher dose Z-drugs (Table 5) and higher dose benzodiazepines (Additional file 5 table S6).

Z-drug users were more likely prescribed a new antipsychotic (HR 2.37, 95\% CI 1.84-3.04) or antidepressant (HR 2.32, 95\% CI 1.65-3.25) during follow-up compared to non-users with sleep disturbance (Table 4). There was a small increase in antibiotic prescribing subsequent to new Z-drug prescription compared to non-users with proximal GP consultation (HR 1.19, 95\% CI 1.08-1.30). Rates of new prescribing were generally similar post-Zdrug prescription to post-benzodiazepine prescription and were greater with increasing Z-drug dose (Table 5).

\section{Additional analyses}

Similar associations to those for time-varying prescribed dose were observed when analysing the (non-time-varying) average number of doses prescribed over the exposure period (Additional file 5 
Table 2 Adjusted hazard ratios for new Z-drug prescription and adverse events for people with dementia

\begin{tabular}{|c|c|c|c|c|}
\hline \multirow[t]{2}{*}{ Outcome } & \multirow[b]{2}{*}{$\begin{array}{l}\text { Z-drug } \\
(n=3532)\end{array}$} & \multicolumn{3}{|l|}{ Comparator } \\
\hline & & $\begin{array}{l}\text { Sleep disturbance, no } \\
\text { sedative-hypnotic }(n=1833)\end{array}$ & $\begin{array}{l}\text { No Z-drug, proximal GP } \\
\text { consultation }(n=10,214)\end{array}$ & $\begin{array}{l}\text { Benzodiazepine } \\
(n=5172)\end{array}$ \\
\hline \multicolumn{5}{|l|}{ Fracture } \\
\hline Incidence rate per 100PY (events) & $11.4(164)$ & $7.6(130)$ & $8.4(269)$ & $12.5(223)$ \\
\hline Age, sex-adjusted HR ( $95 \% \mathrm{Cl}$ ) & NA & $1.39(1.08-1.78)$ & $1.40(1.14-1.70)$ & $0.93(0.76-1.14)$ \\
\hline Fully adjusted HR $(95 \% \mathrm{Cl})^{\mathrm{a}}$ & NA & $1.32(0.99-1.75)$ & $1.34(1.08-1.67)^{\mathrm{b}}$ & $0.99(0.80-1.23)$ \\
\hline \multicolumn{5}{|l|}{ Hip fracture } \\
\hline Incidence rate per 100PY (events) & $5.7(84)$ & $3.4(60)$ & $3.5(115)$ & $5.1(94)$ \\
\hline Age, sex-adjusted HR (95\% Cl) & NA & $1.53(1.07-2.18)$ & $1.64(1.23-2.19)$ & $1.11(0.82-1.49)$ \\
\hline Fully adjusted HR $(95 \% \mathrm{Cl})^{\mathrm{a}}$ & NA & $1.38(0.92-2.06)$ & $1.59(1.15-2.19)^{b}$ & $1.10(0.87-1.65)$ \\
\hline \multicolumn{5}{|l|}{ Forearm/wrist/hand fracture } \\
\hline Incidence rate per 100PY (events) & $2.0(29)$ & $1.1(20)$ & $1.5(48)$ & $2.5(46)$ \\
\hline Age, sex-adjusted HR (95\% Cl) & NA & $1.80(0.95-3.41)$ & $1.35(0.85-2.14)$ & $0.81(0.51-1.28)$ \\
\hline Fully adjusted HR $(95 \% \mathrm{Cl})^{\mathrm{a}}$ & NA & $1.44(0.60-3.47)$ & $1.33(0.77-2.31)$ & $1.00(0.59-1.70)$ \\
\hline \multicolumn{5}{|l|}{ Fall } \\
\hline Incidence rate per 100PY (events) & $37.1(473)$ & $27.3(384)$ & $25.8(767)$ & $35.8(585)$ \\
\hline Age, sex-adjusted HR (95\% Cl) & NA & $1.12(0.97-1.29)$ & $1.52(1.36-1.71)$ & $1.06(0.94-1.20)$ \\
\hline Fully adjusted HR $(95 \% \mathrm{Cl})^{\mathrm{a}}$ & NA & $1.02(0.87-1.21)$ & $1.43(1.26-1.62)^{b}$ & $1.08(0.95-1.22)$ \\
\hline \multicolumn{5}{|l|}{ Mortality } \\
\hline Incidence rate per 100PY (events) & $28.4(436)$ & $16.7(301)$ & $24.1(799)$ & $39.0(736)$ \\
\hline Age, sex-adjusted HR (95\% Cl) & NA & $1.51(1.29-1.77)$ & $1.20(1.06-1.34)$ & $0.72(0.64-0.81)$ \\
\hline Fully adjusted HR $(95 \% \text { Cl) })^{a}$ & NA & $1.38(1.14-1.66)^{b}$ & $1.08(0.94-1.23)$ & $0.73(0.64-0.83)^{b}$ \\
\hline \multicolumn{5}{|l|}{ Acute bacterial infection } \\
\hline Incidence rate per 100PY (events) & $47.8(416)$ & $43.6(220)$ & $40.6(1325)$ & $57.8(371)$ \\
\hline Age, sex-adjusted HR (95\% Cl) & NA & $1.13(0.94-1.37)$ & $1.24(1.09-1.42)$ & $1.01(0.86-1.18)$ \\
\hline Fully adjusted HR $(95 \% \text { Cl) })^{a}$ & NA & $1.02(0.82-1.27)$ & $1.13(0.98-1.31)$ & $0.92(0.78-1.10)$ \\
\hline \multicolumn{5}{|c|}{ Ischaemic stroke/transient ischaemic attack } \\
\hline Incidence rate per 100PY (events) & $6.2(93)$ & $4.4(77)$ & $5.5(178)$ & $6.0(110)$ \\
\hline Age, sex-adjusted HR (95\% Cl) & NA & $1.32(0.95-1.83)$ & $1.20(0.94-1.54)$ & $1.03(0.78-1.36)$ \\
\hline Fully adjusted HR $(95 \% \mathrm{Cl})^{\mathrm{a}}$ & NA & $1.35(0.90-2.04)$ & $1.14(0.86-1.50)$ & $1.05(0.78-1.43)$ \\
\hline \multicolumn{5}{|l|}{ Venous thromboembolism } \\
\hline Incidence rate per 100PY (events) & $1.5(22)$ & $1.3(21)$ & $1.4(43)$ & $2.5(47)$ \\
\hline Age, sex-adjusted HR (95\% Cl) & NA & $1.64(0.97-2.79)$ & $1.14(0.74-1.76)$ & $0.79(0.50-1.25)$ \\
\hline Fully adjusted HR $(95 \% \mathrm{Cl})^{\mathrm{a}}$ & NA & $1.65(0.74-3.69)^{c}$ & $1.12(0.67-1.85)$ & $0.82(0.50-1.34)$ \\
\hline
\end{tabular}

Abbreviations: $H R$ hazard ratio, $C l$ confidence interval, GP general practitioner, $P Y$ person-years

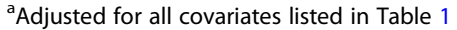

${ }^{b}$ Fully adjusted HR remaining statistically significant after controlling the false discovery rate to < $5 \%$ (based on 13 outcomes)

${ }^{c}$ Not adjusted for antiepileptics, antiplatelet drugs, pneumonia vaccine, and anxiety symptoms due to model instability

table S7-S8). In the sensitivity analysis using a comparator of those with sleep disturbance and no associated mention of $>6 \mathrm{~h}$ sleep, associations for Z-drug use were generally slightly reduced (Additional file 5 tables S9-S10). Finally, associations for Z-drug use compared to the 1601 patients prescribed benzodiazepines for sleep disturbance were very similar to when comparing to any benzodiazepine (Additional file 5, table S11).

\section{Discussion}

We found evidence of increased risks of falls, fractures, and ischaemic stroke in people with dementia prescribed $\mathrm{Z}$-drugs at higher doses. The associations observed were similar or greater in magnitude to those for higher dose benzodiazepine prescription. One in six Z-drug prescriptions were commenced at higher doses of equivalent to $7.5 \mathrm{mg}$ zopiclone daily or greater. We did not detect any consistent or clinically significant increased risks of 
Table 3 Adjusted hazard ratios for Z-drug prescription and adverse events for people with dementia according to prescribed daily defined dose of Z-drugs

\begin{tabular}{|c|c|c|c|c|c|}
\hline \multirow{2}{*}{$\begin{array}{l}\text { Outcome } \\
\text { and daily } \\
\text { defined } \\
\text { dose } \\
\text { prescribed }^{\text {a }}\end{array}$} & \multirow{2}{*}{$\begin{array}{l}\text { No. } \\
\text { events } \\
\text { in the } \\
\text { Z-drug } \\
\text { cohort }\end{array}$} & \multicolumn{2}{|c|}{ Sleep disturbance and no sedative-hypnotic $(n=1833)$} & \multicolumn{2}{|c|}{ No Z-drug, proximal GP consultation $(n=10,214)$} \\
\hline & & Age, sex adjusted & Fully adjusted $^{b}$ & Age, sex adjusted & Fully adjusted $^{\mathbf{b}}$ \\
\hline \multicolumn{6}{|l|}{ Fracture } \\
\hline$\leq 0.5$ & 117 & $1.30(0.99-1.70)$ & $1.22(0.90-1.66)$ & $1.33(1.07-1.65)$ & $1.28(1.01-1.63)$ \\
\hline $0.6-0.9$ & 5 & $1.28(0.51-3.20)$ & $1.06(0.39-2.89)$ & $1.24(0.50-3.05)$ & $1.08(0.42-2.76)$ \\
\hline$\geq 1$ & 42 & $1.70(1.19-2.42)$ & $1.67(1.13-2.46)^{c}$ & $1.66(1.18-2.34)$ & $1.58(1.09-2.28)^{c}$ \\
\hline \multicolumn{6}{|l|}{ Hip fracture } \\
\hline$\leq 0.5$ & 55 & $1.30(0.88-1.93)$ & $1.21(0.78-1.90)$ & $1.42(1.03-1.97)$ & $1.43(1.00-2.06)$ \\
\hline $0.6-0.9$ & $<5$ & $1.10(0.26-4.67)$ & $0.79(0.15-4.11)$ & $1.27(0.31-5.22)$ & $1.05(0.24-4.64)$ \\
\hline$\geq 1$ & 27 & $2.30(1.45-3.65)$ & $1.96(1.16-3.31)^{c}$ & $2.50(1.61-3.89)$ & $2.36(1.44-3.87)^{c}$ \\
\hline \multicolumn{6}{|c|}{ Forearm fracture } \\
\hline$\leq 0.5$ & 20 & $1.66(0.81-3.40)$ & $1.22(0.48-3.12)$ & $1.28(0.77-2.15)$ & $1.29(0.73-2.27)$ \\
\hline$\geq 0.6$ & 9 & $2.18(0.98-4.85)$ & $1.91(0.67-5.47)$ & $1.53(0.72-3.24)$ & $1.42(0.59-3.38)$ \\
\hline \multicolumn{6}{|l|}{ Fall } \\
\hline$\leq 0.5$ & 335 & $1.05(0.90-1.22)$ & $0.95(0.80-1.13)$ & $1.43(1.26-1.63)$ & $1.35(1.17-1.56)^{c}$ \\
\hline $0.6-0.9$ & 14 & $0.84(0.49-1.44)$ & $0.73(0.41-1.29)$ & $1.19(0.70-2.02)$ & $1.07(0.61-1.87)$ \\
\hline$\geq 1$ & 124 & $1.42(1.15-1.74)$ & $1.33(1.06-1.66)^{\mathrm{c}}$ & $1.92(1.58-2.35)$ & $1.81(1.46-2.34)^{c}$ \\
\hline \multicolumn{6}{|l|}{ Mortality } \\
\hline$\leq 0.5$ & 321 & $1.49(1.26-1.77)$ & $1.38(1.14-1.68)^{c}$ & $1.18(1.03-1.34)$ & $1.07(0.93-1.24)$ \\
\hline $0.6-0.9$ & 16 & $1.56(0.95-2.56)$ & $1.60(0.96-2.70)$ & $1.25(0.77-2.02)$ & $1.22(0.75-1.99)$ \\
\hline$\geq 1$ & 99 & $1.56(1.24-1.96)$ & $1.33(1.03-1.71)$ & $1.26(1.02-1.55)$ & $1.06(0.85-1.33)$ \\
\hline \multicolumn{6}{|c|}{ Acute bacterial infection } \\
\hline$\leq 0.5$ & 297 & $1.15(0.98-1.36)$ & $1.00(0.83-1.20)$ & $1.37(1.20-1.57)$ & $1.23(1.06-1.43)^{c}$ \\
\hline $0.6-0.9$ & 13 & $0.98(0.56-1.72)$ & $0.86(0.47-1.57)$ & $1.20(0.69-2.08)$ & $1.12(0.63-1.99)$ \\
\hline$\geq 1$ & 106 & $1.40(1.12-1.76)$ & $1.25(0.98-1.60)$ & $1.64(1.32-2.04)$ & $1.52(1.21-1.91)^{c}$ \\
\hline \multicolumn{6}{|c|}{ Ischaemic stroke/transient ischaemic attack } \\
\hline$\leq 0.5$ & 55 & $1.04(0.72-1.51)$ & $1.12(0.71-1.75)$ & $0.96(0.71-1.30)$ & $0.95(0.68-1.31)$ \\
\hline $0.6-0.9$ & 5 & $1.97(0.78-4.96)$ & $1.98(0.74-5.28)$ & $1.74(0.72-4.19)$ & $1.71(0.74-3.98)$ \\
\hline$\geq 1$ & 33 & $2.07(1.36-3.15)$ & $1.88(1.14-3.10)^{\mathrm{c}}$ & $1.90(1.30-2.79)$ & $1.61(1.08-2.42)$ \\
\hline \multicolumn{6}{|c|}{ Venous thromboembolism } \\
\hline$\leq 0.5$ & 26 & $2.01(1.16-3.49)$ & $2.00(0.90-4.47)$ & $1.35(0.85-2.13)$ & $1.26(0.74-2.12)$ \\
\hline$\geq 0.6$ & $<5$ & $0.78(0.27-2.25)$ & $0.85(0.21-3.39)$ & $0.56(0.21-1.54)$ & $0.66(0.22-1.95)$ \\
\hline
\end{tabular}

Abbreviations: $H R$ hazard ratio, $\mathrm{Cl}$ confidence interval, GP general practitioner

${ }^{2}$ The reference group for all comparisons is no Z-drug prescription. Most patients assigned to the '0.6-0.9 DDD' Z-drug group were prescribed 3.75 mg zopiclone with instructions similar to 'TAKE ONE OR TWO AT NIGHT'

${ }^{\mathrm{b}}$ Adjusted for all covariates listed in Table 1

${ }^{c}$ Fully adjusted HR remaining statistically significant after controlling the false discovery rate to < $5 \%$ (based on 37 tests; 11 outcomes with three dose categories and two outcomes with two dose categories)

mortality, infection, or venous thromboembolism with Z-drug use. PlwD prescribed higher dose Z-drugs were also more likely to be admitted to hospital, visit their GP, and be further prescribed antipsychotics, antidepressants, and antibiotics.

We designed the study to minimise possible sources of bias [34]. Although we were unable to measure dementia severity, we adjusted for the duration since the dementia diagnosis, prescription of dementia medications and antipsychotics, history of agitation/psychosis, and end of life care. However, there may be residual confounding by dementia severity for some comparisons. Although underlying severity was unclear, the mortality results suggest that, compared to Z-drug users, the sleep 
Table 4 Adjusted hazard and rate ratios for new Z-drug prescription and new prescriptions, GP consultations, and hospital admissions for people with dementia

\begin{tabular}{|c|c|c|c|c|}
\hline \multirow[t]{2}{*}{ Outcome } & \multirow[b]{2}{*}{$\begin{array}{l}\text { Z-drug } \\
(n=3532)\end{array}$} & \multicolumn{3}{|l|}{ Comparator } \\
\hline & & $\begin{array}{l}\text { Sleep disturbance, no sedative- } \\
\text { hypnotic }(n=1833)\end{array}$ & $\begin{array}{l}\text { No Z-drug, proximal GP consultation } \\
(n=10,214)\end{array}$ & $\begin{array}{l}\text { Benzodiazepine } \\
(n=5172)\end{array}$ \\
\hline \multicolumn{5}{|l|}{ Number of hospital admissions } \\
\hline Rate per 100PY (events) & $\begin{array}{l}126.7 \\
(1944)\end{array}$ & $93.0(1671)$ & $107.7(3563)$ & $129.4(2441)$ \\
\hline $\begin{array}{l}\text { Age, sex-adjusted RR } \\
(95 \% \mathrm{Cl})\end{array}$ & NA & $1.34(1.20-1.49)$ & $1.26(1.15-1.39)$ & $0.98(0.89-1.09)$ \\
\hline $\begin{array}{l}\text { Fully adjusted RR (95\% } \\
\text { Cl) }\end{array}$ & NA & $1.26(1.13-1.40)^{b}$ & $1.17(1.07-1.27)^{\mathrm{b}}$ & $0.92(0.84-1.01)$ \\
\hline \multicolumn{5}{|l|}{ Number of GP consultations } \\
\hline Rate per 100PY (events) & $\begin{array}{l}1387.8 \\
(21292)\end{array}$ & 1124.6 (20209) & $1511.7(50021)$ & $1502.6(28335)$ \\
\hline $\begin{array}{l}\text { Age, sex-adjusted RR } \\
(95 \% \mathrm{Cl})\end{array}$ & NA & $1.29(1.22-1.37)$ & $0.97(0.93-1.01)$ & $0.94(0.90-0.98)$ \\
\hline $\begin{array}{l}\text { Fully adjusted RR (95\% } \\
(\mathrm{Cl})^{\mathrm{a}}\end{array}$ & NA & $1.17(1.12-1.23)^{b}$ & $1.07(1.04-1.11)^{\mathrm{b}}$ & $0.96(0.93-1.00)$ \\
\hline \multicolumn{5}{|l|}{ New antipsychotic prescription } \\
\hline $\begin{array}{l}\text { Incidence rate per 100PY } \\
\text { (events) }\end{array}$ & 38.7 (331) & $10.3(130)$ & $15.2(227)$ & $75.4(532)$ \\
\hline $\begin{array}{l}\text { Age, sex-adjusted HR } \\
(95 \% \mathrm{Cl})\end{array}$ & NA & $2.53(2.03-3.14)$ & $3.68(3.10-4.37)$ & $0.79(0.68-0.90)$ \\
\hline $\begin{array}{l}\text { Fully adjusted HR (95\% } \\
(\mathrm{Cl})^{\mathrm{a}}\end{array}$ & NA & $2.37(1.84-3.04)^{b}$ & $3.85(3.18-4.65)^{\mathrm{b}}$ & $0.86(0.74-0.99)$ \\
\hline \multicolumn{5}{|c|}{ New antidepressant prescription } \\
\hline $\begin{array}{l}\text { Incidence rate per 100PY } \\
\text { (events) }\end{array}$ & $23.8(172)$ & $8.2(82)$ & $10.3(199)$ & $30.3(242)$ \\
\hline $\begin{array}{l}\text { Age, sex-adjusted HR } \\
(95 \% \mathrm{Cl})\end{array}$ & NA & $2.24(1.70-2.94)$ & $2.52(2.05-3.10)$ & $0.86(0.71-1.05)$ \\
\hline $\begin{array}{l}\text { Fully adjusted HR (95\% } \\
(\mathrm{Cl})^{\mathrm{a}}\end{array}$ & NA & $2.32(1.65-3.25)^{b}$ & $2.65(2.09-3.37)^{b}$ & $0.88(0.71-1.10)$ \\
\hline \multicolumn{5}{|l|}{ New antibiotic prescription } \\
\hline $\begin{array}{l}\text { Incidence rate per 100PY } \\
\text { (events) }\end{array}$ & $109.1(791)$ & $61.2(517)$ & 89.5 (1738) & $111.0(1096)$ \\
\hline $\begin{array}{l}\text { Age, sex-adjusted HR } \\
(95 \% \mathrm{Cl})\end{array}$ & NA & $1.46(1.30-1.65)$ & $1.27(1.17-1.38)$ & $1.01(0.92-1.10)$ \\
\hline $\begin{array}{l}\text { Fully adjusted HR }(95 \% \\
\text { CI) }\end{array}$ & NA & $1.34(1.17-1.52)^{b}$ & $1.19(1.08-1.30)^{b}$ & $1.05(0.95-1.15)$ \\
\hline
\end{tabular}

Abbreviations: $H R$ hazard ratio, $C l$ confidence interval, $P Y$ person-years, $R R$ rate ratio

${ }^{a}$ Adjusted for all covariates listed in Table 1

${ }^{\mathrm{b}}$ Fully adjusted HR remaining statistically significant after controlling the false discovery rate to $<5 \%$ (based on 13 outcomes)

disturbance group not prescribed sedatives may have less severe dementia, however that the non-users with proximal GP consultation had comparable dementia severity. Residual confounding by sleep disturbance severity is also a possibility. Sleep disturbance was challenging to identify within the electronic primary care record as highlighted in our validation study, where only $42 \%$ of our selected 'sleep disturbance' patients had sleep disturbance confirmed by their GP practice. This could be partly due to the sometimes contradictory 'sleep pattern' records or that the sleep disturbance recorded was transient or due to other causes, such as urinary incontinence or alcohol abuse. The comparable fall risk in the Z-drug and 'sleep disturbance' group could be due to urinary incontinence and alcohol use being more common in the 'sleep disturbance' group. The coding of 'sleep disturbance' was often vague and may represent conditions other than insomnia. It may be that some of the 'sleep disturbance and no sedative' group had milder cases of sleep disturbance than those prescribed Zdrugs. Performing a sensitivity analysis on the sleep disturbance definition reduced our effects slightly. Residual 
Table 5 Adjusted hazard ratios for Z-drug prescription and new prescriptions, GP consultations, and hospital admissions for people with dementia according to prescribed daily defined doses (DDDs) of Z-drugs

\begin{tabular}{|c|c|c|c|c|c|}
\hline \multirow{2}{*}{$\begin{array}{l}\text { Outcome } \\
\text { and daily } \\
\text { defined } \\
\text { dose } \\
\text { prescribed }^{\text {a }}\end{array}$} & \multirow{2}{*}{$\begin{array}{l}\text { No. } \\
\text { events } \\
\text { in the } \\
\text { Z-drug } \\
\text { cohort }\end{array}$} & \multicolumn{2}{|c|}{ No sedative-hypnotic, sleep disturbance $(n=1833)$} & \multicolumn{2}{|c|}{ No Z-drug, proximal GP consultation $(n=10,214)$} \\
\hline & & Age, sex adjusted & Fully adjusted $^{\mathbf{b}}$ & Age, sex adjusted & Fully adjusted $^{\mathbf{b}}$ \\
\hline
\end{tabular}

Number of hospital admissions ${ }^{c}$

$\begin{array}{llllll}\leq 0.5 & 1403 & 1.24(1.10-1.39) & 1.18(1.05-1.32) & 1.19(1.07-1.31) & 1.10(1.00-1.20) \\ 0.6-0.9 & 63 & 1.06(0.76-1.49) & 1.22(0.88-1.69) & 1.03(0.74-1.43) & 1.10(0.80-1.51) \\ \geq 1 & 472 & 1.35(1.15-1.58) & 1.29(1.10-1.50)^{d} & 1.32(1.15-1.52) & 1.22(1.06-1.39)^{d}\end{array}$

Number of GP consultations ${ }^{c}$

$\begin{array}{lllllr}\leq 0.5 & 9230 & 1.21(1.14-1.29) & 1.10(1.05-1.16)^{d} & 0.89(0.85-0.94) & 1.00(0.96-1.04) \\ 0.6-0.9 & 690 & 1.12(0.96-1.32) & 1.10(0.95-1.26) & 0.83(0.71-0.97) & 1.02(0.88-1.17) \\ \geq 1 & 4823 & 1.23(1.12-1.34) & 1.16(1.08-1.25)^{d} & 0.91(0.84-0.98) & 1.06(0.99-1.13)\end{array}$

Incident antipsychotic prescription

$\begin{array}{llllll}\leq 0.5 & 214 & 2.08(1.64-2.62) & 2.00(1.54-2.61)^{d} & 3.09(2.56-3.74) & 3.31(2.69-4.07)^{d} \\ 0.6-0.9 & 18 & 3.35(2.04-5.50) & 2.75(1.57-4.81)^{d} & 5.15(3.19-8.32) & 4.82(2.81-8.25)^{d} \\ \geq 1 & 99 & 4.05(3.10-5.31) & 3.56(2.61-4.85)^{d} & 5.81(4.55-7.42) & 5.82(4.44-7.63)^{d}\end{array}$

Incident antidepressant prescription

$\begin{array}{llllll}\leq 0.5 & 124 & 1.25(1.14-1.37) & 1.16(1.05-1.28)^{d} & 2.47(1.91-3.19) & 2.39(1.90-2.99)^{d} \\ 0.6-0.9 & 8 & 1.20(0.83-1.73) & 1.13(0.76-1.68) & 2.67(1.19-5.99) & 3.00(1.49-6.01) \\ \geq 1 & 40 & 1.37(1.17-1.61) & 1.31(1.11-1.54)^{d} & 3.47(2.32-5.18) & 2.93(2.04-4.19)^{d}\end{array}$

Incident antibiotic prescription

$\begin{array}{llllll}\leq 0.5 & 583 & 1.43(1.27-1.63) & 1.30(1.13-1.49)^{d} & 1.25(1.14-1.37) & 1.16(1.05-1.28)^{d} \\ 0.6-0.9 & 27 & 1.36(0.93-2.00) & 1.20(0.79-1.81) & 1.20(0.83-1.73) & 1.13(0.76-1.68) \\ \geq 1 & 181 & 1.57(1.32-1.88) & 1.46(1.21-1.76)^{d} & 1.37(1.17-1.61) & 1.31(1.11-1.54)^{d}\end{array}$

Abbreviations: $H R$ hazard ratio, $C l$ confidence interval, GP general practitioner

${ }^{a}$ The reference group for all comparisons is no Z-drug prescription. Most patients assigned to the '0.6-0.9 DDD' Z-drug group were prescribed 3.75 mg zopiclone with instructions similar to 'TAKE ONE OR TWO AT NIGHT'

${ }^{\mathrm{b}}$ Adjusted for all covariates listed in Table 1

${ }^{\mathrm{C}}$ Estimates provided are rate ratios (95\% confidence intervals)

${ }^{d}$ Fully adjusted HR remaining statistically significant after controlling the false discovery rate to $<5 \%$ (based on 37 tests; 11 outcomes with three dose categories and two outcomes with two dose categories)

confounding by severity of insomnia or dementia could also affect the associations with higher Z-drug doses. We also had no data on genetic information and environmental factors, which may influence fall risk in people living with dementia [35]. There was likely some small residual confounding due to new admission to a care home; however, we were unable to accurately ascertain the admission date to control for this.

Our study was strengthened by additional comparisons to non-users with a proximal GP visit and new benzodiazepine users. As benzodiazepines are also prescribed for anxiety and behavioural disturbances of dementia, there may be residual confounding by dementia and sleep disturbance severity in the comparison between Z-drug and benzodiazepine users. However, when instead restricting to benzodiazepines likely prescribed for sleep disturbance, our findings were very similar. Dosing instructions were often missing for benzodiazepines, and although we made plausible assumptions based on the complete prescriptions, some misclassification of exposures is possible. Recording of prescriptions issued in primary care is accurate; however, we lacked data on medications prescribed in secondary care or obtained elsewhere. Medication adherence is unknown; therefore, the Z-drug effects may be underestimated if many patients prescribed Z-drugs had not taken them. Studies report high positive predictive values for patients coded with our study outcomes in UK primary care data [36-41]. Potential under-reporting in CPRD was improved through linkage to HES and ONS. However, we likely underestimated forearm fracture incidence as many do not require hospital admission. Similarly, GP records of falls may under-represent all falls that occur in the older population, but more accurately represent 'injurious falls requiring medical attention' [42]. Our study was strengthened by using a new-user design and careful selection and followup of patients taking Z-drugs alone and not concurrent 
with other sedative-hypnotics [43, 44]. Our findings are generalizable to most people with diagnosed dementia and sleep disturbance. Few patients were prescribed zaleplon or zolpidem and none eszopiclone; however, as they exert the same pharmacological action as zopiclone, the adverse effects of these agents are likely similar.

\section{Comparison with other studies \\ Fractures}

Few studies have examined Z-drug dose and fracture risk. Greater risks of hip fracture were reported among older US care home residents taking higher dose Zdrugs, although limited by small numbers [45]. Additionally, few studies have examined fracture risk in PlwD taking Z-drugs. Consistent with our findings, a study of hospital records of PlwD in Japan reported increased fracture risks with Z-drug use, but they were unable to ascertain whether the prescription was given before or after the fracture [46]. Various studies report associations between Z-drugs and fracture risk, and specifically hip fracture risk, in older adults $[12,13]$. However, our study and others suggest this relative risk is lower in PlwD [47, 48]. For example, in US nursing home residents, greater odds ratios were estimated between non-benzodiazepine hypnotic drug use and hip fracture in residents with no or only mild cognitive impairment than with moderate-severe cognitive impairment [47]. Z-drugs likely increase fracture risk through their effects on gait and balance $[49,50]$. A randomised trial reported more tandem walk failures upon nighttime awakening among older adults randomised to $5 \mathrm{mg}$ zolpidem compared to placebo [51].

\section{Falls}

Z-drugs were originally claimed to cause fewer falls than benzodiazepines [52]; however, we found similar or larger effects. This is consistent with findings from older men in the Osteoporotic Fractures in Men study [53]. More fall-related injuries were observed with Z-drug use in older people in Taiwan, with greater frequencies when prescribed $>0.6$ DDDs [54]. Increased fall rates have also been observed with dose increases in sedative-hypnotics in nursing home residents with dementia [55].

\section{Cardiovascular outcomes}

Similar to us, more strokes were observed in the Medicine use and Alzheimer's disease (MEDALZ) cohort prescribed Z-drugs and adults in Taiwan prescribed zolpidem $[15,56]$. Unfortunately, neither study estimated dose-specific risks. Mechanisms for Z-drugs causing increased stroke risk are uncertain, but could relate to decreased local cerebral blood flow [56]. However, as prolonged sleep disturbances likely increase stroke risk, residual confounding by sleep disturbance severity and duration could underlie reported associations [57].

\section{Infections}

Analysis of RCT data, generally in younger adults, indicated possible 1.5-2-fold increased infection risks when taking zopiclone and zolpidem [58]. We found inconsistent evidence of increased bacterial infection risk with higher dose Z-drugs. Other studies in older adults, including a MEDALZ cohort study, report no association between Z-drug use and risk of pneumonia [59-61]. Together, these suggest that if acute infection risk increases with higher dose Z-drug use in PlwD, then it is likely to be small, and our study was underpowered to detect it.

\section{Mortality}

Consistent with our findings, a MEDALZ cohort study found benzodiazepine use associated with increased mortality, but not Z-drugs [62]. Studies on Z-drug or benzodiazepine use and mortality in adults have been conflicting, and reported associations may simply stem from increased usage of benzodiazepines with approaching death [63].

\section{Healthcare utilisation}

We observed greater subsequent initiation of antipsychotics and antidepressants among Z-drug users, similarly observed by older people prescribed Z-drugs in a Taiwan study [54]. This likely reflects the increased behavioural and psychological symptoms of dementia as it progresses. The increased hospital visits we observed post-Z-drug initiation could partly reflect the increased fracture and stroke rates in these patients. The Taiwan study also reported greater rates of fall-related injuries requiring hospitalisation among Z-drug users [54].

\section{Conclusions}

Higher doses of Z-drugs should be avoided in PlwD due to increased fracture and stroke risks. One in six PlwD in our study was commenced at $7.5 \mathrm{mg}$ zopiclone or equivalent daily. Prescribers should use the lowest effective dose in the elderly and use simple specific drug regimens, and this advice needs implementing in national guidelines $[64,65]$. Our findings suggest that the safety profile of Z-drugs should be considered similar to benzodiazepines in PlwD. Although the risks associated with low-dose Z-drugs were small, as the effectiveness of Zdrugs is also unproven in dementia, we advise adhering to the Beers criteria guidelines of avoiding Z-drug use in PlwD, where possible [66]. Alternative strategies should be sought for sleep disturbance other than Z-drug or benzodiazepine dose escalation. Where pharmacological management of sleep disturbance is initiated, fracture risk management plans are implemented, and 
prescriptions regularly reviewed to mitigate potential adverse health outcomes. This gives a clear and important steer for the use of hypnotics in people with dementia in clinical practice. This evidence is currently particularly important as social isolation related to the COVID-19 pandemic may increase the frequency of neuropsychiatric symptoms in dementia [67] and limit resources to offer non-pharmacological management approaches.

\section{Supplementary information}

Supplementary information accompanies this paper at https://doi.org/10. 1186/s12916-020-01821-5.

Additional file 1. Read codes to define dementia in CPRD, excluded patients and sleep disturbance.

Additional file 2. Dementia and sleep disturbance validation study. Additional file 3. Read codes in CPRD and ICD-10 codes in HES and ONS to define the outcomes.

Additional file 4. Study protocol registration, Definition of covariates and Multiple Imputation Methods.

Additional file 5. Additional exclusion criteria, frequency of missing data, dose changes during follow-up, additional analysis results tables and flowchart of patient selection.

\section{Abbreviations}

ATC: Anatomical Therapeutic Chemical; BMl: Body mass index; CPRD: Clinical Practice Research Datalink; DDD: Defined daily doses; GP: General practitioner; HES: Hospital Episode Statistics; ICD-10: International classification of diseases 10th revision; MEDALZ: Medicine use and Alzheimer's disease; NNH: Number needed to harm; ONS: Office of National Statistics; PlwD: People living with dementia; PPI: Patient and Public Involvement; TCA: Tricyclic antidepressant

\section{Acknowledgements}

We thank our PPI and healthcare professional advisory group members for their valuable contributions to the study including Kate Massey, Peter Richmond, Lesley Evans, Heather Edwards, Tris Jackson, Mandi Bowhill, Amander Wellings, Rebecca Harmston, Dr. Jay Foden, Dr. Maureen Tilford, Daisy Lo, Magda Turczyn, and Liz Yaxley. Kate Massey and Mandi Bowhill were also members of the study steering committee. We also thank the PPI group coordinator Rhianna Broadway (Inspire, Norfolk and Suffolk NHS Foundation Trust) and the healthcare professional advisory group coordinator Sarah Housden (UEA) for their support during the study. We are grateful to the general practitioners who completed the GP questionnaires as part of the validation study.

\section{Authors' contributions}

$K R$, YKL, CF, IM, GMS, NS, AA, CB, and RH conceived and developed the initial study. KR and GMS drafted the statistical analysis plan. KR, NS, IM, CF, RH, and YKL developed the code lists. KR conducted the statistical analysis and wrote the first manuscript draft, and all authors contributed to the interpretation of findings and revision of the manuscript. The authors read and approved the final manuscript.

\section{Funding}

This research was funded and commissioned by the National Institute of Health Research (NIHR) under their Health Technology Assessment (HTA) programme (NIHR HTA: 14/221/02). KR received additional support from the NIHR East of England Collaboration for Leadership in Applied Health Research \& Care. RH is supported by the UCLH NIHR BRC. The funders had no role in the design of the study, analysis, or interpretation of the findings. The views and opinions expressed are those of the authors and do not necessarily reflect those of the National Health Service, NIHR, or the Department of Health.

\section{Availability of data and materials}

This study is based on data from the Clinical Practice Research Datalink obtained under licence from the UK Medicines and Healthcare products Regulatory Agency (MHRA). However, the interpretation and conclusions contained in this report are those of the authors alone. Linked data from the Clinical Practice Research Datalink is available directly from CPRD. Full code lists for the covariates are available from the corresponding author at kathryn.richardson@uea.ac.uk.

\section{Ethics approval and consent to participate}

The study was approved by the Independent Scientific Advisory Committee for CPRD for Clinical Practice Research Datalink research (protocol 16_181). No further ethical approval was required for the analysis of the data. The CPRD Group has obtained ethical approval from a multi-centre research ethics committee for all purely observational research using CPRD data.

\section{Consent for publication}

Not applicable

\section{Competing interests}

The authors declare no support from any organisation for the submitted work beyond the NIHR grants listed above; YKL reports personal fees from Thame Pharmaceuticals outside of the submitted work, and CB reports grants and personal fees from Acadia pharmaceutical company and Lundbeck and personal fees from Roche, Otusaka, Novartis, Eli Lilly, and Pfizer; no other relationships or activities that could appear to have influenced the submitted work.

\section{Author details}

${ }^{1}$ Norwich Medical School, University of East Anglia, Norwich NR4 7TJ, UK. ${ }^{2}$ School of Life and Health Sciences, Aston University, Birmingham B4 7ET, UK. ${ }^{3}$ Division of Psychiatry, UCL Division of Psychiatry, University College London, Maple House, 149 Tottenham Court Road, London W1T 7NF, UK. ${ }^{4}$ School of Health Sciences, University of East Anglia, Norwich NR4 7TJ, UK. ${ }^{5}$ Medical School, University of Exeter, Exeter EX1 2LU, UK. ${ }^{6}$ Quadram Institute Bioscience, Norwich Research Park, Norwich NR4 7UQ, UK.

Received: 14 July 2020 Accepted: 20 October 2020

Published online: 24 November 2020

\section{References}

1. Guarnieri B, Adorni F, Musicco M, et al. Prevalence of sleep disturbances in mild cognitive impairment and dementing disorders: a multicenter Italian clinical cross-sectional study on 431 patients. Dement Geriatr Cogn Disord. 2012;33:50-8. https://doi.org/10.1159/000335363.

2. Gitlin LN, Hodgson N, Piersol CV, et al. Correlates of quality of life for individuals with dementia living at home: the role of home environment, caregiver, and patient-related characteristics. Am J Geriatr Psychiatry Off J Am Assoc Geriatr Psychiatry. 2014;22:587-97. https://doi.org/10.1016/j.jagp. 2012.11.005.

3. McCurry SM, Logsdon RG, Teri L, et al. Characteristics of sleep disturbance in community-dwelling Alzheimer's disease patients. J Geriatr Psychiatry Neurol. 1999;12:53-9. https://doi.org/10.1177/089198879901200203.

4. Pollak CP, Perlick D. Sleep problems and institutionalization of the elderly. J Geriatr Psychiatry Neurol. 1991;4:204-10.

5. Ashton H. Guidelines for the rational use of benzodiazepines. When and what to use. Drugs. 1994;48:25-40. https://doi.org/10.2165/00003495199448010-00004.

6. Tannenbaum C, Paquette A, Hilmer S, et al. A systematic review of amnestic and non-amnestic mild cognitive impairment induced by anticholinergic, antihistamine, GABAergic and opioid drugs. Drugs Aging. 2012;29:639-58.

7. Swift CG, Swift MR, Hamley J, et al. Side-effect 'tolerance' in elderly longterm recipients of benzodiazepine hypnotics. Age Ageing. 1984;13:335-43. https://doi.org/10.1093/ageing/13.6.335

8. Brett J, Murnion B. Management of benzodiazepine misuse and dependence. Aust Prescr. 2015;38:152-5. https://doi.org/10.18773/austprescr. 2015.055 .

9. Woolcott JC, Richardson KJ, Wiens MO, et al. Meta-analysis of the impact of 9 medication classes on falls in elderly persons. Arch Intern Med. 2009;169: 1952-60. https://doi.org/10.1001/archinternmed.2009.357. 
10. Gunja N. The clinical and forensic toxicology of Z-drugs. J Med Toxicol. 2013;9:155-62. https://doi.org/10.1007/s13181-013-0292-0.

11. Glass J, Lanctôt KL, Herrmann N, et al. Sedative hypnotics in older people with insomnia: meta-analysis of risks and benefits. BMJ. 2005;331:1169. https://doi.org/10.1136/bmj.38623.768588.47.

12. Treves N, Perlman A, Kolenberg Geron L, et al. Z-drugs and risk for falls and fractures in older adults-a systematic review and meta-analysis. Age Ageing. 2018;47:201-8. https://doi.org/10.1093/ageing/afx167.

13. Donnelly K, Bracchi R, Hewitt J, et al. Benzodiazepines, Z-drugs and the risk of hip fracture: a systematic review and meta-analysis. Plos One. 2017;12: e0174730. https://doi.org/10.1371/journal.pone.0174730.

14. Huang C-Y, Chou FH-C, Huang Y-S, et al. The association between zolpidem and infection in patients with sleep disturbance. J Psychiatr Res. 2014;54: 116-20. https://doi.org/10.1016/j.jpsychires.2014.03.017.

15. Huang W-S, Tsai C-H, Lin C-C, et al. Relationship between zolpidem use and stroke risk: a Taiwanese population-based case-control study. J Clin Psychiatry. 2013;74:e433-8. https://doi.org/10.4088/JCP.12m08181.

16. Parsaik AK, Mascarenhas SS, Khosh-Chashm D, et al. Mortality associated with anxiolytic and hypnotic drugs-a systematic review and meta-analysis. Aust N Z J Psychiatry. 2015:0004867415616695. https://doi.org/10.1177/ 0004867415616695.

17. Givens JL, Sanft TB, Marcantonio ER. Functional recovery after hip fracture: the combined effects of depressive symptoms, cognitive impairment, and delirium. J Am Geriatr Soc. 2008;56:1075-9. https://doi.org/10.1111/j.15325415.2008.01711.x

18. McCleery J, Cohen DA, Sharpley AL. Pharmacotherapies for sleep disturbances in dementia. Cochrane Database Syst Rev Published Online First: 2016. doi:https://doi.org/10.1002/14651858.CD009178.pub3.

19. Gustafsson M, Karlsson S, Gustafson Y, et al. Psychotropic drug use among people with dementia - a six-month follow-up study. BMC Pharmacol Toxicol. 2013;14:56. https://doi.org/10.1186/2050-6511-14-56.

20. Sivertsen B, Omvik S, Pallesen S, et al. Cognitive behavioral therapy vs zopiclone for treatment of chronic primary insomnia in older adults: a randomized controlled trial. JAMA. 2006;295:2851-8. https://doi.org/10.1001/ jama.295.24.2851.

21. Herrett E, Gallagher AM, Bhaskaran K, et al. Data resource profile: clinical practice research datalink (CPRD). Int J Epidemiol. 2015;44:827-36. https:// doi.org/10.1093/ije/dyv098

22. Chisholm J. The Read clinical classification. Br Med J. 1990;300:1092. https:// doi.org/10.1136/bmj.300.6732.1092.

23. Herbert A, Wijlaars L, Zylbersztejn A, et al. Data resource profile: Hospital Episode Statistics Admitted Patient Care (HES APC). Int J Epidemiol. 2017;46: 1093-1093i. https://doi.org/10.1093/ije/dyx015.

24. Patel V. Deaths registered in England and Wales: 2017. London: Office for National Statistics; 2018.

25. Department for Communities and Local Government, UK Government. English indices of deprivation 2015. https://www.gov.uk/government/ statistics/english-indices-of-deprivation-2015. Accessed 22 Oct 2019.

26. Lawlor DA, Tilling K, Davey SG. Triangulation in aetiological epidemiology. Int J Epidemiol. 2016;45:1866-86. https://doi.org/10.1093/ije/dyw314.

27. Rogers W. Regression standard errors in clustered samples. Stata Tech Bull 1994;3.https://econpapers.repec.org/article/tsjstbull/y_3a1994_3av_3a3_3 ai_3a13_3asg17.htm. Accessed 11 Dec 2019.

28. Grambsch PM, Therneau TM. Proportional hazards tests and diagnostics based on weighted residuals. Biometrika. 1994;81:515-26. https://doi.org/10. 1093/biomet/81.3.515.

29. Groenwold RHH, Klungel OH, Altman DG, et al. Adjustment for continuous confounders: an example of how to prevent residual confounding. CMAJ Can Med Assoc J. 2013;185:401-6. https://doi.org/ 10.1503/cmaj.120592.

30. De Laet C, Kanis JA, Odén A, et al. Body mass index as a predictor of fracture risk: a meta-analysis. Osteoporos Int. 2005;16:1330-8. https://doi.org/ 10.1007/s00198-005-1863-y.

31. Altman DG, Andersen PK. Calculating the number needed to treat for trials where the outcome is time to an event. BMJ. 1999;319:1492-5.

32. White IR, Royston P, Wood AM. Multiple imputation using chained equations: issues and guidance for practice. Stat Med. 2011;30:377-99. https://doi.org/10.1002/sim.4067.

33. Benjamini $Y$, Hochberg $Y$. Controlling the false discovery rate: a practical and powerful approach to multiple testing. J R Stat Soc Ser B Methodol. 1995;57:289-300. https://doi.org/10.2307/2346101.
34. Sterne JA, Hernán MA, Reeves $B C$, et al. ROBINS-I: a tool for assessing risk of bias in non-randomised studies of interventions. BMJ. 2016;355:14919. https://doi.org/10.1136/bmj.i4919.

35. Sharma $S$, Mueller $C$, Stewart $R$, et al. Predictors of falls and fractures leading to hospitalization in people with dementia: a representative cohort study. J Am Med Dir Assoc. 2018;19:607-12. https://doi.org/10.1016/j.jamda.2018.03. 009.

36. Khan NF, Harrison SE, Rose PW. Validity of diagnostic coding within the General Practice Research Database: a systematic review. Br I Gen Pract. 2010;60:e128-36. https://doi.org/10.3399/bjgp10X483562.

37. Staa T-PV, Abenhaim L. The quality of information recorded on a UK database of primary care records: a study of hospitalizations due to hypoglycemia and other conditions. Pharmacoepidemiol Drug Saf. 1994;3: 15-21. https://doi.org/10.1002/pds.2630030106.

38. Huerta C, Johansson $\mathrm{S}$, Wallander $\mathrm{M}$, et al. Risk factors and short-term mortality of venous thromboembolism diagnosed in the primary care setting in the United Kingdom. Arch Intern Med. 2007;167:935-43. https:// doi.org/10.1001/archinte.167.9.935.

39. Ruigómez A, Martín-Merino E, Rodríguez LAG. Validation of ischemic cerebrovascular diagnoses in the health improvement network (THIN). Pharmacoepidemiol Drug Saf. 2010;19:579-85. https://doi.org/10.1002/pds. 1919

40. Gibbs RG, Newson R, Lawrenson R, et al. Diagnosis and initial management of stroke and transient ischemic attack across UK health regions from 1992 to 1996: experience of a national primary care database. Stroke. 2001;32: 1085-90. https://doi.org/10.1161/01.str.32.5.1085.

41. Derby LE, Myers MW, Jick H. Use of dexfenfluramine, fenfluramine and phentermine and the risk of stroke. Br J Clin Pharmacol. 1999;47:565-9. https://doi.org/10.1046/j.1365-2125.1999.00928.x.

42. Gribbin J, Hubbard R, Smith C, et al. Incidence and mortality of falls amongst older people in primary care in the United Kingdom. QJM. 2009; 102:477-83. https://doi.org/10.1093/qjmed/hcp064.

43. Ray WA. Evaluating medication effects outside of clinical trials: new-user designs. Am J Epidemiol. 2003;158:915-20. https://doi.org/10.1093/aje/ kwg231.

44. Schneeweiss S, Patrick AR, Stürmer T, et al. Increasing levels of restriction in pharmacoepidemiologic database studies of elderly and comparison with randomized trial results. Med Care. 2007:45:S131-42. https://doi.org/10.1097/ MLR.0b013e318070c08e.

45. Dore DD, Zullo AR, Mor V, et al. Age, sex, and dose effects of nonbenzodiazepine hypnotics on hip fracture in nursing home residents. J Am Med Dir Assoc. 2018;19:328-332.e2. https://doi.org/10.1016/j.jamda.2017. 09.015.

46. Tamiya $\mathrm{H}$, Yasunaga $\mathrm{H}$, Matusi $\mathrm{H}$, et al. Hypnotics and the occurrence of bone fractures in hospitalized dementia patients: a matched case-control study using a national inpatient database. Plos One. 2015;10:e0129366. https://doi.org/10.1371/journal.pone.0129366.

47. Berry SD, Lee Y, Cai S, et al. Nonbenzodiazepine sleep medication use and hip fractures in nursing home residents. JAMA Intern Med. 2013;173:754-61. https://doi.org/10.1001/jamainternmed.2013.3795.

48. Tolppanen A-M, Taipale $\mathrm{H}$, Tanskanen A, et al. Comparison of predictors of hip fracture and mortality after hip fracture in community-dwellers with and without Alzheimer's disease - exposure-matched cohort study. BMC Geriatr. 2016;16:204. https://doi.org/10.1186/s12877-016-0383-2.

49. Finkle WD, Der JS, Greenland S, et al. Risk of fractures requiring hospitalization after an initial prescription for zolpidem, alprazolam, lorazepam, or diazepam in older adults. J Am Geriatr Soc. 2011;59:1883-90. https://doi.org/10.1111/j.1532-5415.2011.03591.x.

50. Brandt J, Leong C. Benzodiazepines and Z-drugs: an updated review of major adverse outcomes reported on in epidemiologic research. Drugs RD. 2017;17:493-507. https://doi.org/10.1007/s40268-017-0207-7.

51. Frey DJ, Ortega JD, Wiseman C, et al. Influence of zolpidem and sleep inertia on balance and cognition during nighttime awakening: a randomized placebo-controlled trial. J Am Geriatr Soc. 59:73-81. https://doi. org/10.1111/j.1532-5415.2010.03229.x.

52. Allain $\mathrm{H}$, Bentué-Ferrer $\mathrm{D}$, Polard $\mathrm{E}$, et al. Postural instability and consequent falls and hip fractures associated with use of hypnotics in the elderly: a comparative review. Drugs Aging. 2005;22:749-65.

53. Diem SJ, Ewing SK, Stone KL, et al. Use of non-benzodiazepine sedative hypnotics and risk of falls in older men. J Gerontol Geriatr Res. 2014;3:158. https://doi.org/10.4172/2167-7182.1000158. 
54. Yu N-W, Chen P-J, Tsai H-J, et al. Association of benzodiazepine and Z-drug use with the risk of hospitalisation for fall-related injuries among older people: a nationwide nested case-control study in Taiwan. BMC Geriatr. 2017;17:140. https://doi.org/10.1186/s12877-017-0530-4.

55. Sterke CS, van Beeck EF, van der Velde N, et al. New insights: dose-response relationship between psychotropic drugs and falls: a study in nursing home residents with dementia. J Clin Pharmacol. 2012;52:947-55. https://doi.org/ 10.1177/0091270011405665.

56. Taipale $\mathrm{H}$, Koponen $\mathrm{M}$, Tanskanen $\mathrm{A}$, et al. Use of benzodiazepines and related drugs is associated with a risk of stroke among persons with Alzheimer's disease. Int Clin Psychopharmacol. 2017;32:135-41. https://doi. org/10.1097/YIC.0000000000000161.

57. Hermann DM, Bassetti CL. Role of sleep-disordered breathing and sleepwake disturbances for stroke and stroke recovery. Neurology. 2016;87:140716. https://doi.org/10.1212/WNL.0000000000003037.

58. Joya FL, Kripke DF, Loving RT, et al. Meta-analyses of hypnotics and infections: eszopiclone, ramelteon, zaleplon, and zolpidem. J Clin Sleep Med JCSM Off Publ Am Acad Sleep Med. 2009:5:377-83.

59. Nakafero G, Sanders RD, Nguyen-Van-Tam JS, et al. The association between benzodiazepines and influenza-like illness-related pneumonia and mortality: a survival analysis using UK Primary Care data. Pharmacoepidemiol Drug Saf 2016;"n/a-n/a. doi:https://doi.org/10.1002/pds.4028.

60. Wang M-T, Wang $\mathrm{Y}-\mathrm{H}$, Chang $\mathrm{H}-\mathrm{A}$, et al. Benzodiazepine and Z-drug use and risk of pneumonia in patients with chronic kidney disease: a population-based nested case-control study. Plos One. 2017;12:e0179472. https://doi.org/10.1371/journal.pone.0179472.

61. Taipale H, Tolppanen A-M, Koponen M, et al. Risk of pneumonia associated with incident benzodiazepine use among community-dwelling adults with Alzheimer disease. CMAJ. 2017;189:E519-29. https://doi.org/10.1503/cmaj. 160126.

62. Saarelainen L, Tolppanen A-M, Koponen M, et al. Risk of death associated with new benzodiazepine use among persons with Alzheimer disease: a matched cohort study. Int J Geriatr Psychiatry. 2018;33:583-90. https://doi. org/10.1002/gps.4821.

63. Neutel $\mathrm{Cl}$, Johansen HL. Association between hypnotics use and increased mortality: causation or confounding? Eur J Clin Pharmacol. 2015;71:637-42. https://doi.org/10.1007/s00228-015-1841-z

64. Walley T, Scott AK. Prescribing in the elderly. Postgrad Med J. 1995;71:466-71.

65. Milton JC, Hill-Smith I, Jackson SHD. Prescribing for older people. BMJ. 2008; 336:606-9. https://doi.org/10.1136/bmj.39503.424653.80.

66. By the 2019 American Geriatrics Society Beers Criteria® Update Expert Panel. American Geriatrics Society 2019 Updated AGS Beers Criteria ${ }^{\circledR}$ for potentially inappropriate medication use in older adults. J Am Geriatr Soc 2019;67:674694. doi:https://doi.org/10.1111/jgs.15767.

67. Brown EE, Kumar S, Rajji TK, et al. Anticipating and mitigating the impact of the COVID-19 pandemic on Alzheimer's disease and related dementias. Am J Geriatr Psychiatry Published Online First: 18 April 2020. doi:https://doi.org/ 10.1016/j.jagp.2020.04.010.

\section{Publisher's Note}

Springer Nature remains neutral with regard to jurisdictional claims in published maps and institutional affiliations.

Ready to submit your research? Choose BMC and benefit from:
- fast, convenient online submission
- thorough peer review by experienced researchers in your field
- rapid publication on acceptance
- support for research data, including large and complex data types
- gold Open Access which fosters wider collaboration and increased citations
- maximum visibility for your research: over 100M website views per year
At BMC, research is always in progress.
Learn more biomedcentral.com/submissions

